\section{A) Check for updates}

Cite this: Org. Biomol. Chem., 2021, 19, 2502

Received 23rd December 2020, Accepted 25th February 2021

DOI: 10.1039/d0ob02567d rsc.li/obc

\title{
C4-aldehyde of guaiazulene: synthesis and derivatisation $\uparrow$
}

\author{
Georgia E. Williams, (DD a Gabriele Kociok-Köhn, (ID) b Tony D. James (ID a,c and \\ Simon E. Lewis iD *a
}

\begin{abstract}
Guaiazulene is an alkyl-substituted azulene available from natural sources and is a much lower cost starting material for the synthesis of azulene derivatives than azulene itself. Here we report an approach for the selective functionalisation of guaiazulene which takes advantage of the acidity of the protons on the guaiazulene C4 methyl group. The aldehyde produced by this approach constitutes a building block for the construction of azulenes substituted on the seven-membered ring. Derivatives of this aldehyde synthesised by alkenylation, reduction and condensation are reported, and the halochromic properties of a subset of these derivatives have been studied.
\end{abstract}

\section{Introduction}

Azulene (1) is a non-alternant bicyclic aromatic hydrocarbon, that has significantly different properties to those of its isomer naphthalene (2). ${ }^{1}$ Whereas the HOMO-LUMO gap in naphthalene leads to absorption in the UV region, the corresponding molecular orbitals are closer in energy in azulene, hence leading to an absorption in the visible region. Thus, naphthalene is colourless but azulene is blue. Azulene also displays anomalous fluorescence characteristics, being an exception to Kasha's rule, with the $\mathrm{S}_{2} \rightarrow \mathrm{S}_{0}$ transition being the dominant mode of emission. ${ }^{2}$ Additional interesting optical properties such as halochromism, ${ }^{3}$ solvatochromism ${ }^{4}$ and barochromism $^{5}$ have also been reported for certain azulenes. Azulene also has a dipole moment of $1.08 \mathrm{D}$, unusually high for a simple hydrocarbon. ${ }^{6}$ This arises from the contribution of resonance structure $\mathbf{1}^{\prime}$, in which both individual rings are $6 \pi$ aromatic systems (Fig. 1). These notable properties have led to azulenes being used extensively as both colorimetric ${ }^{7}$ and fluorescent ${ }^{8}$ probes for various analytes. Azulenes have also been employed in solar cells ${ }^{9}$ and organic electronics, ${ }^{10}$ as well as in medicinal chemistry. ${ }^{11}$

These various applications have been enabled by the development of synthetic routes to substituted azulenes. ${ }^{12}$ One

\footnotetext{
${ }^{a}$ Department of Chemistry, University of Bath, Bath, BA2 7AY, UK.

E-mail: s.e.lewis@bath.ac.uk

${ }^{b}$ Materials and Chemical Characterisation $\left(M C^{2}\right)$, University of Bath, Bath, BA2 $7 A Y$, $U K$

${ }^{c}$ School of Chemistry and Chemical Engineering, Henan Normal University, Xinxiang 453007, P. R. China

$\dagger$ Electronic supplementary information (ESI) available. CCDC 2047319. For ESI and crystallographic data in CIF or other electronic format see DOI: 10.1039/ d0ob02567d
}

approach is to synthesise the azulene ring from a precursor which already incorporates the desired substituents, for example by aromatisation of 1,1-dicyanodihydroazulenes ${ }^{13}$ or by a carbene insertion/ring expansion approach. ${ }^{14}$ Another strategy is to employ synthetic methodology that introduces the desired substituents onto a pre-existing azulene ring. Approaches for functionalisation of the 5-membered ring of azulene include $\mathrm{S}_{\mathrm{E}} \mathrm{Ar}$ reactions, ${ }^{15}$ cross-coupling ${ }^{16}$ and $\mathrm{C}-\mathrm{H}$ activation. ${ }^{17}$ Approaches for functionalisation of the 7-membered ring of azulene are less numerous, but include vicarious nucleophilic substitution ${ }^{18}$ and nucleophilic addition/ rearomatisation. ${ }^{16 g, 19}$ However, regardless of the diversity of approaches for azulene derivatisation, work in this area is hindered by the high cost of azulene itself - preparation of the parent hydrocarbon requires multistep procedures and/or reagents that are themselves costly. ${ }^{15 a, 20}$ There is therefore a motivation to use an alternative azulene starting material which is abundantly available from natural sources and hence has a much lower cost, namely guaiazulene (3).

Guaiazulene and its derivatives are sesquiterpene natural products produced by various organisms, in particular gorgonian octocorals and the fungal genus Lactarius. ${ }^{21}$ Moreover, guaiazulene may be produced from the essential oils isolated from genera such as Eucalyptus, ${ }^{22}$ Matricaria (chamomile) ${ }^{23}$ and others. ${ }^{24}$ These processes proceed via the dehydration/
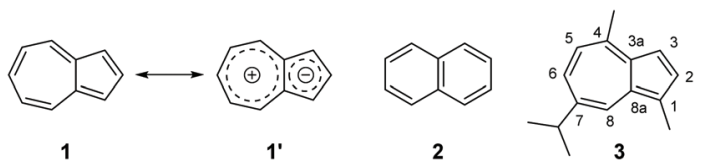

Fig. 1 Structures of azulene, naphthalene and guaiazulene. 
dehydrogenation of other sesquiterpenes such as guaiol and occur in high yields, permitting the large scale production of guaiazulene at low cost. The main uses of guaiazulene and its derivative sodium guaiazulene-3-sulfonate are as anti-inflammatory agents and in cosmetics. ${ }^{25}$

The alkyl groups on guaiazulene do not influence the azulene chromophore significantly, but they impart a much lower melting point $\left(30-33^{\circ} \mathrm{C}\right)^{26}$ and guaiazulene can undergo very slow decomposition on exposure to air. ${ }^{27}$ However, derivatives bearing electron withdrawing groups are generally stable. Methods for derivatisation of guaiazulene are not as extensively developed as for azulene itself and in some instances may be hindered by the alkyl substituents. On the other hand, the alkyl substituents of guaiazulene can be seen as handles for further derivatisation themselves. ${ }^{28}$ One strategy to achieve this exploits the known acidity of the protons on the guaiazulene C4 methyl group. ${ }^{3 h, 17 n, 29}$ The low $\mathrm{p} K_{\mathrm{a}}$ of these protons arises from the resonance stabilisation of the corresponding conjugate base, $[3-\mathrm{H}]^{-}$that may be attributed to resonance structure $\left[3^{\prime}-\mathrm{H}\right]^{-}$(Scheme 1$)$ that comprises an aromatic cyclopentadienide substructure.

Amide acetals such as dimethylformamide dimethyl acetal (DMFDMA) are versatile reagents able to effect a variety of synthetic transformations. ${ }^{30}$ Reaction of an amide acetal with aryl methyl groups to give the corresponding enamines was first reported by Meerwein ${ }^{31}$ and was further developed by Bredereck. $^{32}$ Oxidative cleavage of such enamines is possible using sodium periodate (with no requirement for osmium tetroxide). ${ }^{33}$ Taken together, these two procedures allow formation of an aryl aldehyde in two steps from the corresponding aryl methyl group. The reaction mechanism requires that the arene is able to stabilise a benzylic anion, hence electron-poor arene substrates are generally required (Scheme 2a). We reasoned that this synthetic sequence could be applicable to guaiazulene, resulting in a regioselective transformation of the C4 methyl group to an aldehyde, although whether the C4 methyl protons were sufficiently acidic was unclear at the outset. To our knowledge there are three prior reports of azulenyl aldehydes being formed from the corresponding azulene methyl groups in this way. In 2006, Kim, Osuka and coworkers reported the reaction of a 4-methylazulene 4 , bearing an electron-withdrawing ester at the 1-position, which would lower the $\mathrm{p} K_{\mathrm{a}}$ of the $\mathrm{C} 4$ methyl protons (Scheme $2 \mathrm{~b}$ ). ${ }^{34}$ In 2015 , Leino and co-workers reported the reaction of 6-methylazulene 6, which lacks any other substituents (Scheme 2c). ${ }^{35}$ Most recently, in 2018 Shoji and co-workers reported the reaction of various 2-methylazulenes of type $\mathbf{8}$, finding that the reaction

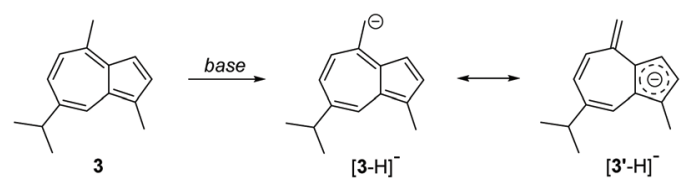

Scheme 1 Guaiazulene deprotonates preferentially at the C4 methyl group.
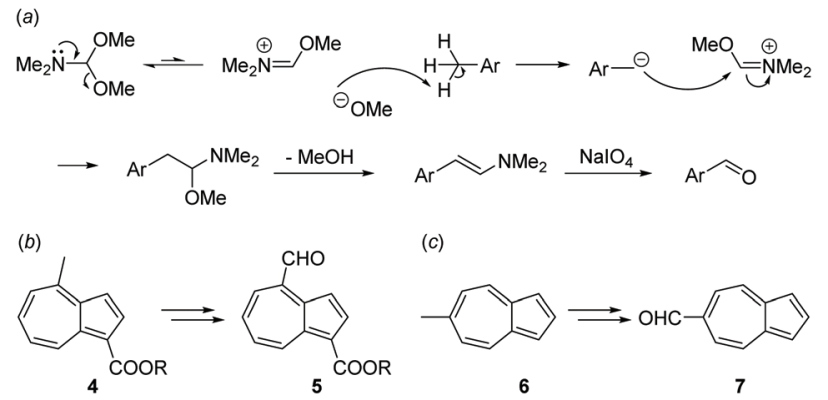

(c)

(d)

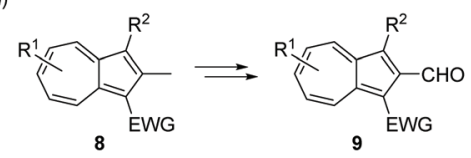

Scheme 2 (a) Mechanism for transformation of aryl methyl group to aryl aldehyde. (b) Work of Kim, Osuka and co-workers. (c) Work of Leino and co-workers. (d) Work of Shoji and co-workers.

did not proceed without an electron-withdrawing group at the 1-position (Scheme 2d). ${ }^{36}$

In this paper we describe the first synthesis of guaiazulene4-carbaldehyde, as well as some further transformations of this compound.

\section{Results and discussion}

Treatment of guaiazulene 3 with DMFDMA in DMF gave crude enamine $\mathbf{1 0}$ as a green solid after aqueous workup. $\$$ This proved to be somewhat unstable and could not be purified by chromatography on silica. Thus, 10 was used crude, and was directly subjected to oxidative cleavage with $\mathrm{NaIO}_{4}$ to give novel aldehyde $\mathbf{1 1}$ in 77\% yield over two steps (Scheme 3). In contrast to 10, aldehyde 11 could be purified by chromatography on silica, and is stable in pure form. Crystals of $\mathbf{1 1}$ were subjected to X-ray diffraction analysis and the resultant structure is shown in Fig. 2.

We anticipate that aldehyde $\mathbf{1 1}$ may find various uses in synthesis, since it is accessible in a concise fashion from a cheap azulenic starting material. Reduction of 11 with $\mathrm{NaBH}_{4}$ furnished alcohol 12 in sufficient purity that chromatography

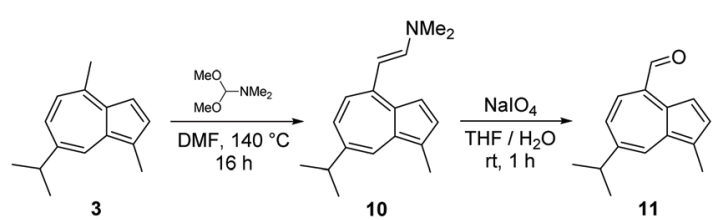

Scheme 3 Synthesis of guaiazulene-4-carbaldehyde 11.

$\$$ Although 10 has not been reported previously, various substituted analogues have been described, having been formed under Vilsmeier conditions - see ref. 41. 


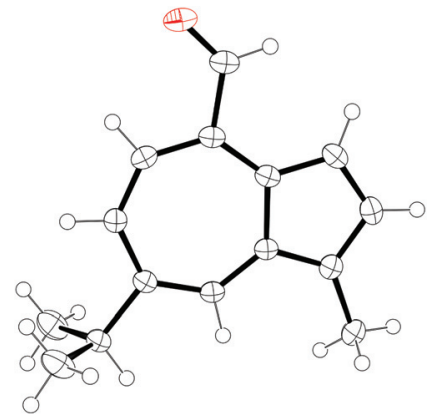

Fig. 2 ORTEP representation of the X-ray structure of 11. Ellipsoids are shown at $50 \%$ probability. Hydrogens are shown as spheres of arbitrary radius. CCDC 2047319.†

was not required (Scheme 4a). Alkenylation of $\mathbf{1 1}$ by HornerWadsworth-Emmons reaction was carried out using a variety of phosphonates (Scheme 4b, Table 1). ${ }^{37}$ Alkenes 20 and 21 were synthesised from phosphonate reagents containing additional Lewis basic groups, and as such the modified Masamune-Rousch reaction conditions were employed in these instances. ${ }^{38}$

C4-alkenylated guaiazulene derivatives such as 14-22 have been reported previously, ${ }^{3 h, 11 j, 29 c j, 29 l-n, 29 r}$ although in these cases the C4 methyl carbon reacted as a nucleophile, as opposed to as an electrophile as is the case here. A notable property of certain C4-alkenylated guaiazulenes described previously is their halochromism. ${ }^{3 h, 29 c}$ Accordingly, selected alkenylated derivatives of $\mathbf{1 1}$ and their response to Brønsted acid were studied. 4-Fluorostyrylazulene 19 was titrated against increasing quantities of trifluoroacetic acid in $\mathrm{CH}_{2} \mathrm{Cl}_{2}$ and $\mathrm{UV} /$ vis absorption spectra were acquired (Fig. 3).

The absorption maxima at $\lambda=289 \mathrm{~nm}$ and $\lambda=319 \mathrm{~nm}$ decreased in intensity with increasing acid concentration, whereas a new absorption maximum at $\lambda=447 \mathrm{~nm}$ (in the blue region) was seen to increase in intensity; an isosbestic point was observed at $\lambda \approx 388 \mathrm{~nm} .{ }^{1} \mathrm{H}$-NMR spectra of 19 in $\mathrm{CDCl}_{3}$ and in $\mathrm{CDCl}_{3}$ /TFA were acquired (Fig. 4), and show the site of protonation to be the guaiazulene C3 position. Specifically, for the spectrum in $\mathrm{CDCl}_{3}$, the $\mathrm{H}-3$ resonance is observed as a $1 \mathrm{H}$ doublet at $7.46 \mathrm{ppm}$, whereas in $\mathrm{TFA} / \mathrm{CDCl}_{3}$

(a)

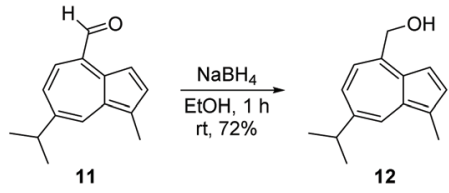

(b)<smiles>[R]C=Cc1ccc(C(C)C)cc2c(C)ccc1-2</smiles>

Scheme 4 (a) Reduction of 11. (b) Horner-Wadsworth-Emmons reaction of 11.
Table 1 Horner-Wadsworth-Emmons reactions of 11

\begin{tabular}{|c|c|c|c|c|c|}
\hline Entry & $\mathrm{R}$ & Product & $\mathrm{R}^{\prime}$ & Method $^{a, b}$ & Yield (\%) \\
\hline 1 & & 14 & Et & A & 38 \\
\hline 2 & & 15 & Et & A & 46 \\
\hline 3 & & 16 & Et & A & 18 \\
\hline 4 & & 17 & Et & A & 56 \\
\hline 5 & & 18 & Et & A & 42 \\
\hline 6 & & 19 & Et & A & 44 \\
\hline 7 & & 20 & $\mathrm{Me}$ & B & 41 \\
\hline 8 & & 21 & Et & B & 33 \\
\hline 9 & & 22 & Et & A & 50 \\
\hline
\end{tabular}

${ }^{a}$ Method A: $60 \%$ NaH (2 eq.), THF, rt, 2 h. ${ }^{b}$ Method B: LiCl (3 eq.), $\mathrm{DBU}(3 \mathrm{eq}),. \mathrm{THF}, 0{ }^{\circ} \mathrm{C}, 3 \mathrm{~h}$.

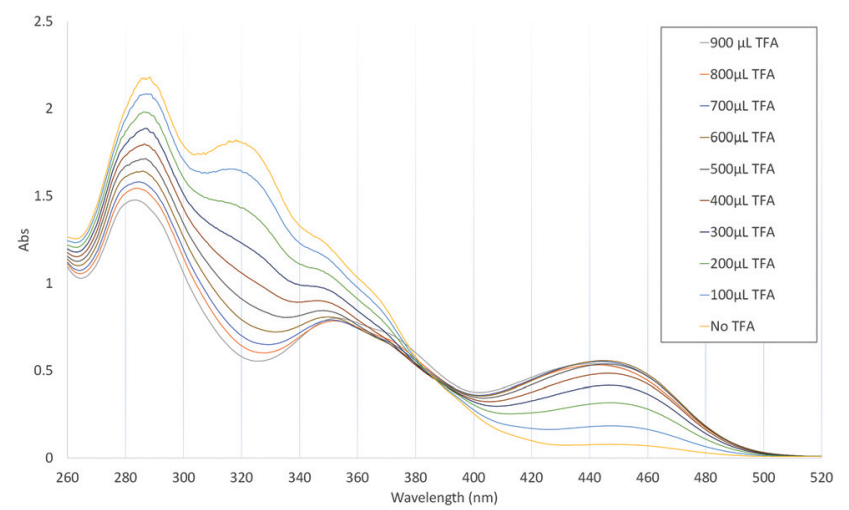

Fig. 3 Titration of $19(0.08 \mathrm{mM})$ with TFA (1 $\mathrm{M}$ in $\left.\mathrm{CH}_{2} \mathrm{Cl}_{2}\right)$ in $\mathrm{CH}_{2} \mathrm{Cl}_{2}$.

it is observed as a $2 \mathrm{H}$ broad singlet at $4.08 \mathrm{ppm}$, this chemical shift being indicative of an $\mathrm{sp}^{3}$-hybridised carbon. Further support for the guaiazulene C3 position being the site of protonation comes from comparison with spectra of similar azulenium cations generated by hydride abstraction from dihydroazulenes. ${ }^{13 d, 39}$

Cyano-substituted 15, chloro-substituted 18 and $\alpha, \beta$-unsaturated ester 20 were investigated in a similar fashion, and all were observed by NMR to undergo protonation at the same position (Fig. S1-S3†). Whereas 15 and 18 exhibited similar maxima and isosbestic points to 19 (Fig. S4 and S5†), the isosbestic point for 20 was blue shifted (Fig. S6†). As a further demonstration of the scope for functionalisation of 11, formation of a nitrone was attempted. Azulenyl nitrones are of 

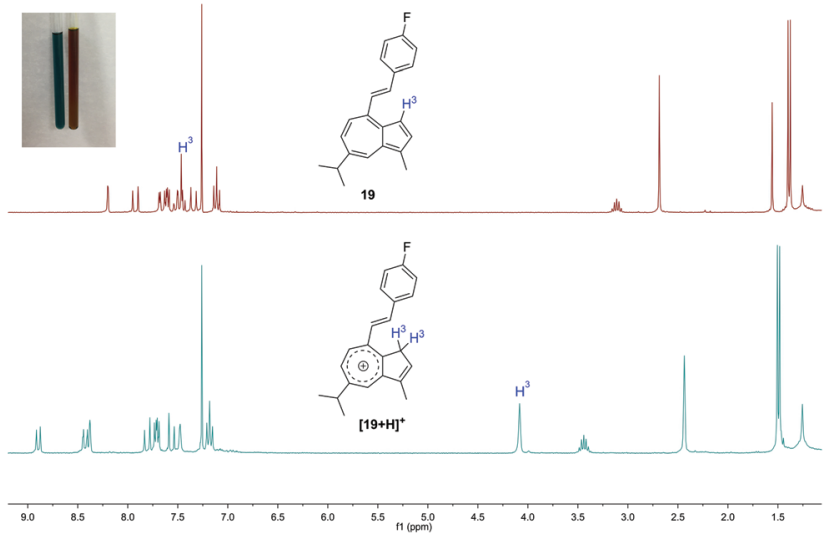

Fig. 4 Top: ${ }^{1} \mathrm{H}-\mathrm{NMR}$ spectrum of 19 in $\mathrm{CDCl}_{3}$. Bottom: ${ }^{1} \mathrm{H}-\mathrm{NMR}$ spectrum of 19 in TFA/CDCl 3 1: 9. Inset: NMR tubes containing 19 in $\mathrm{CDCl}_{3}$ (left) and 19 in TFA/ $\mathrm{CDCl}_{3}$ (right).

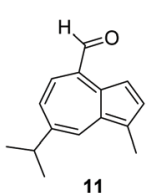

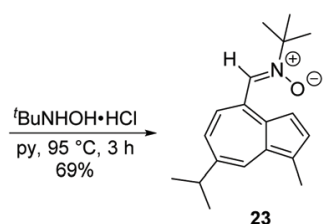

Scheme 5 Synthesis of nitrone 23

interest as free radical spin traps with neuroprotective activity and potential clinical utility. ${ }^{40}$ In the present case, condensation of $\mathbf{1 1}$ with $\mathrm{N}$-tert-butylhydroxylamine gave nitrone $\mathbf{2 3}$ (Scheme 5).

\section{Conclusions}

We have described the concise synthesis of an azulene bearing an aldehyde at the 4-position (11). The use of a low-cost, commercially available azulene starting material and the requirement for only one purification step render $\mathbf{1 1}$ an attractive building block for the synthesis of more complex azulenes bearing substituents at the 4-position. We have demonstrated the utility of $\mathbf{1 1}$ through its use in reduction, alkenylation and condensation reactions.

\section{Experimental}

\section{General details}

Reactions which required the use of anhydrous, inert atmosphere techniques were carried out under an atmosphere of argon. Reaction solvents were obtained by passing though anhydrous alumina columns using an Innovative Technology Inc. PS-400-7 solvent purification system. In most cases, solvents were purchased as "anhydrous" grade from Fisher Scientific. "Petrol" refers to petroleum spirit b.pt. $40-60{ }^{\circ} \mathrm{C}$. TLC was performed using aluminium backed plates pre-coated with Alugram®SIL G/UV $254 \mathrm{~nm}$. Where needed, visualisation was accomplished by UV light and/or $\mathrm{KMnO}_{4}$ or using phosphomolybdic acid (PMA) dip followed by gentle warming. Following work up, the organic layers were routinely dried using anhydrous $\mathrm{MgSO}_{4}$ and evaporated using a Büchi rotary evaporator. When necessary, further drying was facilitated by high vacuum. During purification, flash column chromatography was carried out using 60 angstroms $(\AA)$ silica gel (40-75 micron) purchased from Sigma Aldrich. ${ }^{1} \mathrm{H}$ and ${ }^{13} \mathrm{C}$ NMR spectra were acquired on Agilent ProPulse $500 \mathrm{MHz}$ or Bruker Advance 250, 300, 400 or 500 megahertz (MHz) instruments at $298 \mathrm{~K}$. All spectra were referenced to residual solvent peaks; chemical shifts are reported in parts per million (ppm) relative to residual chloroform $\left(\delta=7.26 \mathrm{ppm},{ }^{1} \mathrm{H} ; 77.16 \mathrm{ppm}\right.$, $\left.{ }^{13} \mathrm{C}\right)$. All ${ }^{13} \mathrm{C}\left\{{ }^{1} \mathrm{H}\right\}$ resonances are singlets, unless stated otherwise. Coupling constants, $J$, reported in $\mathrm{Hz}$, were calculated using MestreNova 9.0 to the nearest $0.1 \mathrm{~Hz}$. The following abbreviations are used to label the multiplicities: s, singlet; d, doublet; t, triplet; q, quartet; quin, quintet; sex, sextet; sep, septet; dd, doublet of doublets; dq, doublet of quartets; td, triplet of doublets; m, multiplet and br, broad. ${ }^{1} \mathrm{H}$ and ${ }^{13} \mathrm{C}\left\{{ }^{1} \mathrm{H}\right\}$ assignments for novel compounds are corroborated though $2 \mathrm{D}$ (COSY, HSQC, HMBC). Infrared (IR) spectra were recorded on a PerkinElmer Spectrum 100 ATR-FTIR spectrometer with only selected absorbances quoted as $\nu$ in $\mathrm{cm}^{-1}$. For mass spectrometry a microTOF electrospray time-of-flight (ESI-TOF) mass spectrometer (Bruker Daltonik GmbH, Bremen, Germany) was used. Data are reported in the form of $m / z$. The observed mass and isotope pattern matched the corresponding theoretical values as calculated from the expected elemental formula. Melting points (mp) were determined on an Stanford Research Systems OptiMelt automated capillary melting point apparatus in open capillary tubes and are uncorrected. X-Ray intensity data for $\mathbf{1 1}$ were collected at $150(2) \mathrm{K}$ on a Rigaku SuperNova Dual EosS2 single crystal diffractometer using monochromated $\mathrm{Cu}-\mathrm{K} \alpha$ radiation $(\lambda=1.54184 \AA)$. Unit cell determination, data collection data reduction and absorption correction were performed using the CrysAlisPro software (version 1.171.40.43a, Rigaku Oxford Diffraction, 2019). The structure was solved with SHELXT and refined by a full-matrix least-squares procedure based on F2 (SHELXL-2018/3). All nonhydrogen atoms were refined anisotropically. Hydrogen atoms were placed onto calculated positions and refined using a riding model.

7-Isopropyl-1-methylazulene-4-carbaldehyde (11). Under an atmosphere of nitrogen, guaiazulene $(0.32 \mathrm{~g}, 1.6 \mathrm{mmol}, 1.0$ eq.) was dissolved in degassed anhydrous DMF $(5.0 \mathrm{~mL})$, to which $N, N$-dimethylformamide dimethyl acetal $(0.43 \mathrm{~mL}$, $3.2 \mathrm{mmol}, 2.0$ eq.) was added. The reaction was heated to $140{ }^{\circ} \mathrm{C}$ and stirred for $9 \mathrm{~h}$, during which the solution turned from blue to green. The solution was cooled to room temperature and diluted with ethyl acetate $(30 \mathrm{~mL})$. The organic mixture was washed with water $(30 \mathrm{~mL})$ and $5 \% \mathrm{LiCl}_{(\mathrm{aq})}$ solution $(2 \times 30 \mathrm{~mL})$. The organic phase was dried over $\mathrm{MgSO}_{4}$ and filtered, and the filtrate was concentrated under reduced pressure to give crude 10, used immediately. Under aerobic 
conditions, the crude material was re-dissolved in $\mathrm{THF} / \mathrm{H}_{2} \mathrm{O}$ (30 mL, $1: 1, \mathrm{v} / \mathrm{v})$, to which $\mathrm{NaIO}_{4}(1.0 \mathrm{~g}, 4.8 \mathrm{mmol}, 3.0$ eq.) was added. The solution was stirred room temperature for 2.5 hours, turning from green to blue, then diluted with ethyl acetate $(30 \mathrm{~mL})$. The organic phase was washed with water $(30 \mathrm{~mL})$ and sat. $\mathrm{NaHCO}_{3(\mathrm{aq})}$ solution $(2 \times 30 \mathrm{~mL})$, for which the initial aqueous extract was orange and the latter extract was colourless. The organic phase was dried over $\mathrm{MgSO}_{4}$ and filtered, and the filtrate was concentrated under reduced pressure. The crude material was purified by silica column chromatography, eluting with EtOAc/petrol $\left(5: 95, R_{\mathrm{f}}=0.50\right)$ to give 7-isopropyl-1-methylazulene-4-carbaldehyde $\mathbf{1 1}(0.21 \mathrm{~g}$, $62 \%)$ as a blue-brown solid. ${ }^{1} \mathrm{H}$ NMR $\left(500 \mathrm{MHz}, \mathrm{CDCl}_{3}\right) \delta 1.40$ $\left(\mathrm{d}, J=6.9 \mathrm{~Hz}, 6 \mathrm{H},-\mathrm{CH}\left(\mathrm{CH}_{3}\right)_{2}\right), 2.71\left(\mathrm{~s}, 3 \mathrm{H}, \mathrm{C} 1-\mathrm{CH}_{3}\right), 3.15$ (hept, $\left.J=6.9 \mathrm{~Hz}, 1 \mathrm{H},-\mathrm{CH}\left(\mathrm{CH}_{3}\right)_{2}\right), 7.56(\mathrm{~d}, J=10.4 \mathrm{~Hz}, 1 \mathrm{H}, \mathrm{C} 5-$ H), $7.63(\mathrm{dd}, J=10.5,1.9 \mathrm{~Hz}, 1 \mathrm{H}, \mathrm{C} 6-\mathrm{H}), 7.92(\mathrm{~d}, J=3.6 \mathrm{~Hz}$, $1 \mathrm{H}, \mathrm{C} 3-\mathrm{H}), 8.08(\mathrm{~d}, J=3.7 \mathrm{~Hz}, 1 \mathrm{H}, \mathrm{C} 2-\mathrm{H}), 8.25(\mathrm{~d}, J=1.3 \mathrm{~Hz}$, $1 \mathrm{H}, \mathrm{C} 8-\mathrm{H}), 10.77$ (s, $1 \mathrm{H},-\mathrm{CHO}) \mathrm{ppm} .{ }^{13} \mathrm{C}$ NMR $(500 \mathrm{MHz}$, $\left(\mathrm{CDCl}_{3}\right) \delta 13.0,24.6,39.0,112.4,123.8,126.2,133.9,134.1$, 134.7, 136.2, 141.4, 141.6, 145.1, $195.1 \mathrm{ppm}$. IR $\nu_{\max } 701,761$, 1682, 2861, 2924, $2955 \mathrm{~cm}^{-1}$. Melting point $81-84{ }^{\circ} \mathrm{C}$. HRMS $\left(\right.$ ESI $\left.^{+}\right)$: calculated for $\mathrm{C}_{15} \mathrm{H}_{16} \mathrm{O}[\mathrm{M}+\mathrm{H}]^{+}$213.1274, found 213.1278.

(7-Isopropyl-1-methylazulen-4-yl)methanol (12). To a solution of $11(75 \mathrm{mg}, 0.3 \mathrm{mmol}, 1.0$ eq.) in EtOH was added $\mathrm{NaBH}_{4}(27 \mathrm{mg}, 0.7 \mathrm{mmol}, 2.0$ eq.). The solution was stirred for $1 \mathrm{~h}$ at $\mathrm{rt}$ and was then quenched with water $(10 \mathrm{~mL})$ and extracted with $\mathrm{CH}_{2} \mathrm{Cl}_{2}(3 \times 10 \mathrm{~mL})$, dried over $\mathrm{MgSO}_{4}$ and filtered, then the filtrate was evaporated under reduced pressure to give (7-isopropyl-1-methylazulen-4-yl)methanol as a turquoise gum (54 mg, $72 \%) .{ }^{1} \mathrm{H}$ NMR $\left(500 \mathrm{MHz},\left(\mathrm{CD}_{3}\right)_{2} \mathrm{CO}\right) \delta$ $1.37\left(\mathrm{~d}, J=6.9 \mathrm{~Hz}, 6 \mathrm{H},-\mathrm{CH}\left(\mathrm{CH}_{3}\right)_{2}\right), 2.65\left(\mathrm{~s}, 3 \mathrm{H}, \mathrm{C} 1-\mathrm{CH}_{3}\right), 3.14$ (hept, $\left.J=6.9 \mathrm{~Hz}, 1 \mathrm{H},-\mathrm{CH}\left(\mathrm{CH}_{3}\right)_{2}\right), 4.57(\mathrm{t}, J=5.8 \mathrm{~Hz}, 1 \mathrm{H},-\mathrm{OH})$, $5.18\left(\mathrm{~d}, J=5.3 \mathrm{~Hz}, 2 \mathrm{H},-\mathrm{CH}_{2} \mathrm{OH}\right), 7.25(\mathrm{~d}, J=3.7 \mathrm{~Hz}, 1 \mathrm{H}, \mathrm{C} 3-$ $\mathrm{H}), 7.52(\mathrm{~d}, J=10.7 \mathrm{~Hz}, 1 \mathrm{H}, \mathrm{C} 5-\mathrm{H}), 7.62-7.59(\mathrm{~m}, 2 \mathrm{H}, \mathrm{C} 2-\mathrm{H}$, $\mathrm{C} 6-\mathrm{H}), 8.27$ (d, $J=2.0 \mathrm{~Hz}, 1 \mathrm{H}, \mathrm{C} 8-\mathrm{H}) \mathrm{ppm} .{ }^{13} \mathrm{C}$ NMR $\left(500 \mathrm{MHz},\left(\mathrm{CD}_{3}\right)_{2} \mathrm{CO}\right) \delta 12.9,25.0,39.0,64.5,111.3,121.3$, 125.4, 133.9, 135.7, 135.7, 137.2, 138.6, 141.4, 147.4 ppm. IR $\nu_{\max } 1008,1386,2924,2956,3316 \mathrm{~cm}^{-1}$. HRMS (ESI $\left.{ }^{+}\right)$: calculated for $\mathrm{C}_{15} \mathrm{H}_{18} \mathrm{O}[\mathrm{M}+\mathrm{H}]^{+}$215.1430, found 215.1438.

\section{General procedure A for Horner-Wadsworth-Emmons reactions}

To a stirred solution of $60 \% \mathrm{NaH}(11 \mathrm{mg}, 0.3 \mathrm{mmol}, 2.0$ eq.) in anhyd. THF $(3 \mathrm{~mL})$ was added dropwise a solution of the required phosphonate $(0.3 \mathrm{mmol}, 2.0$ eq.) in anhyd. THF $(4 \mathrm{~mL})$ under nitrogen. The mixture was stirred for $10 \mathrm{~min}$ after which a solution of $\mathbf{1 1}(30 \mathrm{mg}, 0.1 \mathrm{mmol}, 1.0 \mathrm{eq}$.) in anhyd. THF $(3 \mathrm{~mL})$ was added dropwise. The mixture was stirred at rt until the starting material was consumed as shown by TLC (approx. $2 \mathrm{~h}$ ). The reaction mixture was diluted with water $(10 \mathrm{~mL})$ and extracted with $\mathrm{CH}_{2} \mathrm{Cl}_{2}(3 \times 30 \mathrm{~mL})$. The combined organic fractions were dried over $\mathrm{MgSO}_{4}$ and filtered, then the filtrate was evaporated under reduced pressure. Purification was achieved by column chromatography to give the desired alkenylazulene.
(E)-4-(4-Iodostyryl)-7-isopropyl-1-methylazulene (14). General procedure A was used with diethyl (4-iodobenzyl)phosphonate $(100 \mathrm{mg})$ to give the product as a green waxy gum $(22 \mathrm{mg}$, $38 \%$ ) following purification via column chromatography using Petrol $\left(100 \%, R_{\mathrm{f}} 0.50\right) .{ }^{1} \mathrm{H}$ NMR $\left(500 \mathrm{MHz},\left(\mathrm{CD}_{3}\right)_{2} \mathrm{CO}\right) \delta 1.38(\mathrm{~d}$, $\left.J=6.9 \mathrm{~Hz}, 6 \mathrm{H},-\mathrm{CH}\left(\mathrm{CH}_{3}\right)_{2}\right), 2.66$ (s, 3H, C1- $\left.\mathrm{CH}_{3}\right), 3.15$ (hept, $J$ $\left.=6.9 \mathrm{~Hz}, 1 \mathrm{H},-\mathrm{CH}\left(\mathrm{CH}_{3}\right)_{2}\right), 7.48\left(\mathrm{~d}, J=16.2 \mathrm{~Hz}, 1 \mathrm{H}, \mathrm{C}_{6} \mathrm{H}_{4}-\right.$ $\mathrm{C} \underline{H}=\mathrm{CH}-), 7.58-7.61(\mathrm{~m}, 5 \mathrm{H}, \mathrm{C} 3-\mathrm{H}, \mathrm{C} 5-\mathrm{H}, \mathrm{C} 6-\mathrm{H}, m-\mathrm{I}-\mathrm{Ar}-\mathrm{H})$, $7.67(\mathrm{~d}, J=3.9 \mathrm{~Hz}, 1 \mathrm{H}, \mathrm{C} 2-\mathrm{H}), 7.80(\mathrm{~d}, J=8.4 \mathrm{~Hz}, 2 \mathrm{H}, o-\mathrm{I}-\mathrm{Ar}-$ H), $8.20\left(\mathrm{~d}, J=16.2 \mathrm{~Hz}, 1 \mathrm{H}, \mathrm{C}_{6} \mathrm{H}_{4}-\mathrm{CH}=\mathrm{CH}_{-}\right), 8.25(\mathrm{~s}, 1 \mathrm{H}, \mathrm{C} 8-$ $\mathrm{H}) \mathrm{ppm} .{ }^{13} \mathrm{C}$ NMR $\left(500 \mathrm{MHz},\left(\mathrm{CD}_{3}\right)_{2} \mathrm{CO}\right) \delta 13.1,24.9,38.9,94.2$, 113.1, 120.8, 126.7, 130.0, 130.9, 133.7, 133.7, 135.6, 137.5, 137.7, 137.8, 138.0, 138.8, 141.0, $142.1 \mathrm{ppm}$. IR $\nu_{\max } 763,950$, $2958 \mathrm{~cm}^{-1}$. HMS $\left(\mathrm{ESI}^{+}\right)$: calc. for $\mathrm{C}_{22} \mathrm{H}_{21} \mathrm{I}[\mathrm{M}+\mathrm{H}]^{+}$413.0761, found 413.0771.

(E)-4-(2-(7-Isopropyl-1-methylazulen-4-yl)vinyl)benzonitrile (15). General procedure A was used with diethyl (4-cyanobenzyl) phosphonate $(72 \mathrm{mg})$ to give the product as a green gum (20 $\mathrm{mg}, 46 \%$ ) following purification via column chromatography using Petrol $\left(100 \%, R_{\mathrm{f}} 0.40\right) .{ }^{1} \mathrm{H}$ NMR $(500 \mathrm{MHz}$, $\left.\left(\mathrm{CD}_{3}\right)_{2} \mathrm{CO}\right) \delta 1.38\left(\mathrm{~d}, J=6.9 \mathrm{~Hz}, 6 \mathrm{H},-\mathrm{CH}\left(\mathrm{CH}_{3}\right)_{2}\right), 2.66(\mathrm{~s}, 3 \mathrm{H}$, C1- $\left.\mathrm{CH}_{3}\right), 3.16$ (hept, $\left.J=6.9 \mathrm{~Hz}, 1 \mathrm{H},-\mathrm{CH}\left(\mathrm{CH}_{3}\right)_{2}\right), 7.59$ (d, $J=$ $\left.15.9 \mathrm{~Hz}, 1 \mathrm{H}, \mathrm{C}_{6} \mathrm{H}_{4}-\mathrm{CH}=\mathrm{CH}-\right), 7.59-7.60(\mathrm{~m}, 2 \mathrm{H}, \mathrm{C} 5-\mathrm{H}, \mathrm{C} 6-\mathrm{H})$, $7.63(\mathrm{~d}, J=3.9 \mathrm{~Hz}, 1 \overline{\mathrm{H}}, \mathrm{C} 3-\mathrm{H}), 7.70(\mathrm{~d}, J=3.9 \mathrm{~Hz}, 1 \mathrm{H}, \mathrm{C} 2-\mathrm{H})$, $7.81(\mathrm{~d}, J=8.4 \mathrm{~Hz}, 2 \mathrm{H}, m-\mathrm{CN}-\mathrm{Ar}-\mathrm{H}), 7.98(\mathrm{~d}, J=8.3 \mathrm{~Hz}, 2 \mathrm{H}, o-$ $\mathrm{CN}-\mathrm{Ar}-\mathrm{H}), 8.28(\mathrm{~s}, 1 \mathrm{H}, \mathrm{C} 8-\mathrm{H}), 8.33\left(\mathrm{~d}, J=16.3 \mathrm{~Hz}, 1 \mathrm{H}, \mathrm{C}_{6} \mathrm{H}_{4}{ }^{-}\right.$ $\mathrm{CH}=\mathrm{CH}-)$ ppm. ${ }^{13} \mathrm{C}$ NMR $\left(500 \mathrm{MHz},\left(\mathrm{CD}_{3}\right)_{2} \mathrm{CO}\right) \delta 13.0,24.9$, $38.9,112.1,113.2,119.4,120.8,126.8,128.7,133.0,133.4$, $133.7,133.9,135.7,137.7,138.1,138.2,141.4,141.6$, 142.7 ppm. IR $\nu_{\max } 738,787,1262,2222,2849,2917$, $2959 \mathrm{~cm}^{-1}$. HRMS $\left(\mathrm{ESI}^{+}\right)$: calc. for $\mathrm{C}_{23} \mathrm{H}_{21} \mathrm{~N}[\mathrm{M}+\mathrm{H}]^{+} 312.1747$, found 312.1753 .

(E)-7-Isopropyl-1-methyl-4-(4-nitrostyryl)azulene (16). General procedure A was used with diethyl (4-nitrobenzyl)phosphonate $(77 \mathrm{mg})$ to give the product as a dark green waxy gum $(8.6 \mathrm{mg}$, $18 \%$ ) following purification via column chromatography using Petrol $\left(100 \%, R_{\mathrm{f}} 0.40\right) .{ }^{1} \mathrm{H}$ NMR $\left(500 \mathrm{MHz}, \mathrm{CDCl}_{3}\right) \delta 1.40(\mathrm{~d}, J=$ $\left.6.9 \mathrm{~Hz}, 6 \mathrm{H},-\mathrm{CH}\left(\mathrm{CH}_{3}\right)_{2}\right), 2.70\left(\mathrm{~s}, 3 \mathrm{H}, \mathrm{C} 1-\mathrm{CH}_{3}\right), 3.13$ (hept, $J=$ $\left.6.9 \mathrm{~Hz}, 1 \mathrm{H},-\mathrm{C} \underline{\mathrm{H}}\left(\mathrm{CH}_{3}\right)_{2}\right), 7.40\left(\mathrm{~d}, J=16.2 \mathrm{~Hz}, 1 \mathrm{H}, \mathrm{C}_{6} \mathrm{H}_{4}{ }^{-}\right.$ $\mathrm{C} \underline{\mathrm{H}}=\mathrm{CH}-), 7.43(\mathrm{~d}, J=10.9 \mathrm{~Hz}, 1 \mathrm{H}, \mathrm{C} 5-\mathrm{H}), 7.45$ (br s, $1 \mathrm{H}, \mathrm{C} 3-$ H), $7.54(\mathrm{dd}, J=10.9,1.9 \mathrm{~Hz}, \mathrm{C} 6-\mathrm{H}), 7.72(\mathrm{~s}, 1 \mathrm{H}, \mathrm{C} 2-\mathrm{H})$, 7.74-7.77 (m, 2H, m-NO $\left.{ }_{2}-\mathrm{Ar}-\mathrm{H}\right), 8.15(\mathrm{~d}, J=16.2 \mathrm{~Hz}, 1 \mathrm{H}$, $\left.\mathrm{C}_{6} \mathrm{H}_{4}-\mathrm{CH}=\mathrm{C} \underline{\mathrm{H}}-\right), 8.23(\mathrm{~d}, J=1.9 \mathrm{~Hz}, 1 \mathrm{H}, \mathrm{C} 8-\mathrm{H}), 8.26-8.30(\mathrm{~m}$, $\left.2 \mathrm{H}, o-\mathrm{NO}_{2}-\mathrm{Ar}-\mathrm{H}\right) .{ }^{13} \mathrm{C}$ NMR $\left(500 \mathrm{MHz},\left(\mathrm{CD}_{3}\right)_{2} \mathrm{CO}\right) \delta$ 13.0, 24.9, $39.0,113.2,120.9,124.8,126.9,128.9,132.6,133.9,134.6$, 135.7, 137.7, 138.2, 138.3, 141.4, 141.6, 144.8, 148.2 ppm. IR $\nu_{\max } 744,1335,1511,1592,2924,2957 \mathrm{~cm}^{-1}$. HRMS $\left(\mathrm{ESI}^{+}\right)$: calc. for $\mathrm{C}_{22} \mathrm{H}_{21} \mathrm{NO}_{2}[\mathrm{M}+\mathrm{H}]^{+} 332.1645$, found 332.1653.

(E)-7-Isopropyl-1-methyl-4-(4-(trifluoromethyl)styryl)azulene (17). General procedure A was used with (4-(trifluoromethyl) benzyl)phosphonate $(74 \mathrm{mg})$ to give the product as a green waxy gum $(25 \mathrm{mg}, 56 \%)$ following purification via column chromatography using Petrol $\left(100 \%, \quad R_{\mathrm{f}} 0.60\right) .{ }^{1} \mathrm{H} \quad \mathrm{NMR}$ $\left(500 \mathrm{MHz},\left(\mathrm{CD}_{3}\right)_{2} \mathrm{CO}\right) \delta 1.38\left(\mathrm{~d}, J=6.9 \mathrm{~Hz}, 6 \mathrm{H},-\mathrm{CH}\left(\mathrm{CH}_{3}\right)_{2}\right)$, $2.66\left(\mathrm{~s}, 3 \mathrm{H}, \mathrm{C} 1-\mathrm{CH}_{3}\right), 3.15$ (hept, $\left.J=7.0 \mathrm{~Hz}, 1 \mathrm{H},-\mathrm{C} \underline{\mathrm{H}}\left(\mathrm{CH}_{3}\right)_{2}\right)$, $7.59\left(\mathrm{~d}, J=16.2 \mathrm{~Hz}, 1 \mathrm{H}, \mathrm{C}_{6} \mathrm{H}_{4}-\mathrm{CH}=\mathrm{CH}-\right), 7.61-7.60(\mathrm{~m}, 2 \mathrm{H}$, 
C5-H, C6-H), 7.62 (d, $J=3.9 \mathrm{~Hz}, \mathrm{C} 3-\mathrm{H}), 7.69$ (d, $J=3.8 \mathrm{~Hz}, 1 \mathrm{H}$, C2-H), 7.76 (d, $\left.J=8.1 \mathrm{~Hz}, 2 \mathrm{H}, m-\mathrm{CF}_{3}-\mathrm{Ar}-\mathrm{H}\right), 7.99$ (d, $J=7.8$ $\left.\mathrm{Hz}, 2 \mathrm{H}, o-\mathrm{CF}_{3}-\mathrm{Ar}-\mathrm{H}\right), 8.27$ (s, $\left.1 \mathrm{H}, \mathrm{C} 8-\mathrm{H}\right), 8.30$ (d, $J=16.2 \mathrm{~Hz}$, $\left.1 \mathrm{H}, \mathrm{C}_{6} \mathrm{H}_{4}-\mathrm{CH}=\mathrm{C} \underline{\mathrm{H}}-\right) .{ }^{13} \mathrm{C}$ NMR $\left(500 \mathrm{MHz},\left(\mathrm{CD}_{3}\right)_{2} \mathrm{CO}\right) \delta 142.2$ $\left(\mathrm{q},{ }^{4} J_{\mathrm{CF}}=1.5 \mathrm{~Hz}\right), 141.8,141.3,138.1,138.1,137.6,135.7$, 133.8, 133.2, 132.9, $130.1\left(\mathrm{q},{ }^{2} J_{\mathrm{CF}}=32.0 \mathrm{~Hz}\right), 128.5,126.8$, $126.5\left(\mathrm{q},{ }^{3} J_{\mathrm{CF}}=3.9 \mathrm{~Hz}\right), 125.4\left(\mathrm{q},{ }^{1} J_{\mathrm{CF}}=270.9 \mathrm{~Hz}\right), 120.9,113.2$, 38.9, 24.9, $13.0 \mathrm{ppm}$. IR $\nu_{\max } 825,1065,1106,1320,1413$, $2960 \mathrm{~cm}^{-1}$. HRMS (ESI ${ }^{+}$): calc. for $\mathrm{C}_{23} \mathrm{H}_{21} \mathrm{~F}_{3}[\mathrm{M}+\mathrm{H}]^{+}$355.1668, found 355.1655 .

(E)-4-(4-Chlorostyryl)-7-isopropyl-1-methylazulene (18). General procedure A was used with diethyl (4-chlorobenzyl)phosphonate $(74 \mathrm{mg})$ to yield a green waxy gum (19 $\mathrm{mg}, 42 \%)$ following purification via column chromatography using Petrol $(100 \%$, $\left.R_{\mathrm{f}} 0.40\right) .{ }^{1} \mathrm{H} \mathrm{NMR}\left(500 \mathrm{MHz},\left(\mathrm{CD}_{3}\right)_{2} \mathrm{CO}\right) \delta 1.38(\mathrm{~d}, J=7.0 \mathrm{~Hz}$, $\left.6 \mathrm{H},-\mathrm{CH}\left(\mathrm{CH}_{3}\right)_{2}\right), 2.66$ (s, 3H, C1- $\left.\mathrm{CH}_{3}\right), 3.15$ (hept, $J=6.8 \mathrm{~Hz}$, $\left.1 \mathrm{H},-\mathrm{CH}\left(\mathrm{CH}_{3}\right)_{2}\right), 7.44-7.48(\mathrm{~m}, 2 \mathrm{H}, m-\mathrm{Cl}-\mathrm{Ar}-\mathrm{H}), 7.54(\mathrm{~d}, J=$ $\left.16.2 \mathrm{~Hz}, 1 \mathrm{H}, \mathrm{C}_{6} \mathrm{H}_{4}-\mathrm{CH}=\mathrm{CH}-\right)$, 7.59-7.60 (m, 2H, C5-H, C6-H), 7.60-7.62 (m, 1H, C3-H), 7.67-7.68 (m, 1H, C2-H), 7.79-7.84 (m, 2H,o-Cl-Ar-H), 8.19 (d, $J=16.2 \mathrm{~Hz}, 1 \mathrm{H}, \mathrm{C}_{6} \mathrm{H}_{4}-\mathrm{CH}=\mathrm{CH}_{-}-$), 8.26 (s, $1 \mathrm{H}, \mathrm{C} 8-\mathrm{H}) \mathrm{ppm} .{ }^{13} \mathrm{C} \mathrm{NMR}\left(500 \mathrm{MHz},\left(\mathrm{CD}_{3}\right)_{2} \mathrm{CO}\right) \delta \overline{13.1}$, 24.9, 38.9, 113.1, 120.7, 126.6, 129.6, 129.7, 130.8, 133.5, 133.7, 134.3, 135.6, 137.1, 137.4, 137.7, 137.8, 141.0, 142.1 ppm. IR $\nu_{\max } 772,817,1011,1091,1490,2923 \mathrm{~cm}^{-1}$. HRMS (ESI ${ }^{+}$): calc. for $\mathrm{C}_{22} \mathrm{H}_{21} \mathrm{Cl}[\mathrm{M}+\mathrm{H}]^{+}$321.1405, found 321.1412.

(E)-4-(4-Fluorostyryl)-7-isopropyl-1-methylazulene

(19). General procedure A was used with diethyl (3-fluorobenzyl) phosphonate $(70 \mathrm{mg}$ ) to give the product as a green waxy gum (19 mg, 44\%) following purification via column chromatography using Petrol $\left(100 \%, R_{\mathrm{f}}\right.$ 0.30). ${ }^{1} \mathrm{H}$ NMR $(500 \mathrm{MHz}$, $\left.\left.\left(\mathrm{CD}_{3}\right)_{2} \mathrm{CO}\right) \delta 1.38\left(\mathrm{~d}, J=6.9 \mathrm{~Hz}, 6 \mathrm{H},-\mathrm{CH}_{\left(\mathrm{CH}_{3}\right.}\right)_{2}\right), 2.66(\mathrm{~s}, 3 \mathrm{H}$, C1- $\mathrm{CH}_{3}$ ), 3.15 (hept, $\left.J=6.9 \mathrm{~Hz}, 1 \mathrm{H},-{ }_{-} \mathrm{CH}\left(\mathrm{CH}_{3}\right)_{2}\right), 7.21\left(\mathrm{dd},{ }^{3} J_{\mathrm{HH}}\right.$ $\left.=8.8 \mathrm{~Hz},{ }^{3} J_{\mathrm{HF}}=8.8 \mathrm{~Hz}, 2 \mathrm{H}, o-\mathrm{F}-\mathrm{Ar}-\mathrm{H}\right), 7.54(\mathrm{~d}, J=16.2 \mathrm{~Hz}, 1 \mathrm{H}$, $\mathrm{C}_{6} \mathrm{H}_{4}-\mathrm{CH}=\mathrm{CH}-$ ), 7.59 (br s, 2H, C5-H, C6-H), 7.60 (d, $J=4.0$ $\mathrm{Hz}, 1 \mathrm{H}, \mathrm{C} 3-\mathrm{H}), 7.66$ (d, $J=4.0 \mathrm{~Hz}, 1 \mathrm{H}, \mathrm{C} 2-\mathrm{H}), 7.85\left(\mathrm{dd},{ }^{3} J_{\mathrm{HH}}=\right.$ $\left.8.6 \mathrm{~Hz},{ }^{4} J_{\mathrm{HF}}=5.5 \mathrm{~Hz}, 2 \mathrm{H}, m-\mathrm{F}-\mathrm{Ar}-\mathrm{H}\right), 8.12(\mathrm{~d}, J=16.2 \mathrm{~Hz}, 1 \mathrm{H}$, $\left.\mathrm{C}_{6} \mathrm{H}_{4}-\mathrm{CH}=\mathrm{CH}-\right), 8.26(\mathrm{~s}, 1 \mathrm{H}, \mathrm{C} 8-\mathrm{H}) \mathrm{ppm} .{ }^{13} \mathrm{C}$ NMR $(500 \mathrm{MHz}$, $\left.\left(\mathrm{CD}_{3}\right)_{2} \mathrm{CO}\right) \delta 13.1,25.0,38.9,113.1,116.5\left(\mathrm{~d},{ }^{2} J_{\mathrm{CF}}=21.8 \mathrm{~Hz}\right)$, 120.8, 126.6, $129.9\left(\mathrm{~d},{ }^{5} J_{\mathrm{CF}}=2.4 \mathrm{~Hz}\right), 130.0\left(\mathrm{~d},{ }^{3} J_{\mathrm{CF}}=8.1 \mathrm{~Hz}\right)$, 133.6, 133.7, $134.8\left(\mathrm{~d},{ }^{4} J_{\mathrm{CF}}=3.3 \mathrm{~Hz}\right), 135.7,137.4,137.7,138.0$, $140.9,142.4,163.7\left(\mathrm{~d},{ }^{1} J_{\mathrm{CF}}=246.5 \mathrm{~Hz}\right) \mathrm{ppm}$. IR $\nu_{\max } 771,1215$, 1505, $2955 \mathrm{~cm}^{-1}$. HRMS $\left(\mathrm{ESI}^{+}\right)$: calc. for $\mathrm{C}_{22} \mathrm{H}_{21} \mathrm{~F}[\mathrm{M}+\mathrm{H}]^{+}$ 305.1700, found 305.1706.

\section{General procedure B for Horner-Wadsworth-Emmons reactions}

To the required phosphonate $(0.1 \mathrm{mmol}, 1.0$ eq. $)$ in anhyd. THF (5 mL) was added 1,8-diazabicyclo[5.4.0]undec-7-ene (60 $\mu \mathrm{L}, 0.4 \mathrm{mmol}, 3.0$ eq.) and lithium chloride (18 mg, $0.4 \mathrm{mmol}, 3.0 \mathrm{eq}$.) under nitrogen. The mixture was stirred for $5 \mathrm{~min}$ after which a solution of 11 (30 mg, $0.1 \mathrm{mmol}, 1.0$ eq.) in anhyd. THF (5 mL) was added dropwise. The mixture was stirred at rt until the starting material was consumed as shown by TLC (approx. $3 \mathrm{~h}$ ). The mixture was then quenched with water $(2 \mathrm{~mL})$ and sat. $\mathrm{NH}_{4} \mathrm{Cl}$. The mixture was then extracted with EtOAC $(3 \times 20 \mathrm{~mL})$ and the combined organic fractions were washed with water $(30 \mathrm{~mL})$ and brine $(30 \mathrm{~mL})$, dried over $\mathrm{MgSO}_{4}$ and filtered, then the filtrate was evaporated under reduced pressure. Purification was achieved by column chromatography to give the desired alkenylazulene.

Methyl (E)-3-(7-isopropyl-1-methylazulen-4-yl)acrylate (20). General procedure B was used with methyl 2-(diethoxyphosphoryl)acetate $(26 \mathrm{mg})$ to give the product as a green waxy gum (15 mg, 41\%) following purification via column chromatography using Petrol/EtOAc (95:5; $\left.R_{\mathrm{f}} \quad 0.5\right) .{ }^{1} \mathrm{H} \quad \mathrm{NMR}$ $\left(400 \mathrm{MHz}, \mathrm{CDCl}_{3}\right) \delta 1.39\left(\mathrm{~d}, J=6.9 \mathrm{~Hz}, 6 \mathrm{H},-\mathrm{CH}\left(\mathrm{CH}_{3}\right)_{2}\right), 2.69(\mathrm{~s}$, $3 \mathrm{H}, \mathrm{C} 1-\mathrm{CH}_{3}$ ), 3.11 (hept, $\left.J=6.8 \mathrm{~Hz}, 1 \mathrm{H},-\mathrm{CH}\left(\mathrm{CH}_{3}\right)_{2}\right), 3.87$ (s, $\left.3 \mathrm{H},-\mathrm{OCH}_{3}\right), 6.65$ (d, $J=15.9 \mathrm{~Hz}, 1 \mathrm{H},-\mathrm{CH}=\mathrm{C} \underline{\mathrm{H}}-\mathrm{COOCH}_{3}$ ), 7.29 (d, $J=10.8 \mathrm{~Hz}, 1 \mathrm{H}, \mathrm{C} 5-\mathrm{H}), 7.46$ (d, $J=3.9 \mathrm{~Hz}, 1 \mathrm{H}, \mathrm{C} 3-\mathrm{H})$, 7.50 (dd, $J=10.8,2.0 \mathrm{~Hz}, 1 \mathrm{H}, \mathrm{C} 6-\mathrm{H}), 7.75$ (d, $J=3.3 \mathrm{~Hz}, 1 \mathrm{H}$, C2-H), 8.21 (d, $J=1.9 \mathrm{~Hz}, 1 \mathrm{H}, \mathrm{C} 8-\mathrm{H}), 8.53$ (d, $J=15.9 \mathrm{~Hz}, 1 \mathrm{H}$, $\left.-\mathrm{CH}=\mathrm{CH}-\mathrm{COOCH}_{3}\right)$ ppm. ${ }^{13} \mathrm{C} \mathrm{NMR}\left(500 \mathrm{MHz}, \mathrm{CDCl}_{3}\right) \delta 13.1$, 24.8, 38.6, 52.1, 112.6, 120.4, 122.8, 126.6, 133.6, 134.9, 137.3, $137.4,138.4,138.5,141.8,145.9,167.3 \mathrm{ppm}$. IR $\nu_{\max } 1018$, 1169, 1273, 1718, $2958 \mathrm{~cm}^{-1}$. HRMS (ESI ${ }^{+}$): calc. for $\mathrm{C}_{18} \mathrm{H}_{20} \mathrm{O}_{2}$ $[\mathrm{M}+\mathrm{H}]^{+}$269.1536, found 269.1535.

(E)-3-(7-Isopropyl-1-methylazulen-4-yl)acrylonitrile (21). General procedure B was used with diethyl (cyanomethyl)phosphonate $(25 \mathrm{mg})$ to give the product as a dark green waxy gum (10 $\mathrm{mg}, 33 \%$ ) following purification via column chromatography using Petrol/EtOAc ( $\left.80: 20 ; R_{\mathrm{f}} 0.6\right) .{ }^{1} \mathrm{H}$ NMR $(500 \mathrm{MHz}$, $\left.\mathrm{CDCl}_{3}\right) \delta 1.38\left(\mathrm{~d}, J=6.9 \mathrm{~Hz}, 6 \mathrm{H},-\mathrm{CH}\left(\mathrm{CH}_{3}\right)_{2}\right), 2.69$ (s, 3H, C1$\mathrm{CH}_{3}$ ), 3.12 (hept, $\left.J=6.8 \mathrm{~Hz}, 1 \mathrm{H},-\mathrm{CH}\left(\mathrm{CH}_{3}\right)_{2}\right), 6.13$ (d, $J=16.6$ $\mathrm{Hz}, 1 \mathrm{H},-\mathrm{CH}=\mathrm{C} \underline{\mathrm{H}}-\mathrm{CN}), 7.15(\mathrm{~d}, J=10.7 \mathrm{~Hz}, 1 \mathrm{H}, \mathrm{C} 5-\mathrm{H}), 7.34$ (d, $J=3.8 \mathrm{~Hz}, 1 \mathrm{H}, \mathrm{C} 3-\mathrm{H}), 7.50$ (dd, $J=10.7,2.0 \mathrm{~Hz}, 1 \mathrm{H}, \mathrm{C} 6-\mathrm{H})$, 7.78 (d, $J=3.7 \mathrm{~Hz}, 1 \mathrm{H}, \mathrm{C} 2-\mathrm{H}), 8.22$ (d, $J=1.9 \mathrm{~Hz}, 1 \mathrm{H}, \mathrm{C} 8-\mathrm{H})$, $8.28(\mathrm{~d}, J=16.7 \mathrm{~Hz}, 1 \mathrm{H},-\mathrm{CH}=\mathrm{CH}-\mathrm{CN}) \mathrm{ppm} .{ }^{13} \mathrm{C} \mathrm{NMR}$ $\left(500 \mathrm{MHz}, \mathrm{CDCl}_{3}\right) \delta 13.1,24.7,38.6,101.3,112.4,118.0,119.4$, $127.2,134.0,134.8,136.4,136.9,137.9,139.1,142.5$, 151.9 ppm. IR $\nu_{\max } 721,787,918,976,1422,2215,2959 \mathrm{~cm}^{-1}$. HMS (ESI ${ }^{-}$): calc. for $\mathrm{C}_{17} \mathrm{H}_{17} \mathrm{~N}[\mathrm{M}-\mathrm{H}]^{-}$234.1288, found 234.1290 .

(E)-3-(7-Isopropyl-1-methylazulen-4-yl)- $N$-methoxy- $N$-methylacrylamide (22). General procedure A was used with diethyl (2(methoxy(methyl)amino)-2-oxoethyl)phosphonate $(68 \mathrm{mg})$ to give the product as a light blue waxy gum (28 mg, 50\%) following purification via column chromatography using Petrol $\left(100 \%, R_{\mathrm{f}} 0.3\right) .{ }^{1} \mathrm{H}$ NMR $\left(500 \mathrm{MHz},\left(\mathrm{CD}_{3}\right)_{2} \mathrm{CO}\right) \delta 1.38(\mathrm{~d}, J=6.9$ $\left.\mathrm{Hz}, 6 \mathrm{H},-\mathrm{CH}\left(\mathrm{CH}_{3}\right)_{2}\right), 2.67$ (s, 3H, C1- $\left.\mathrm{CH}_{3}\right), 3.16$ (hept, $J=6.9$ $\left.\mathrm{Hz}, 1 \mathrm{H},-\mathrm{CH}\left(\mathrm{CH}_{3}\right)_{2}\right), 3.30\left(\mathrm{~s}, 3 \mathrm{H}, \mathrm{CH}_{3}\right), 3.84\left(\mathrm{~s}, 3 \mathrm{H}, \mathrm{CH}_{3}\right), 7.36$ (d, $J=15.7 \mathrm{~Hz}, 1 \mathrm{H},-\mathrm{CH}=\mathrm{C} \underline{\mathrm{H}}-\mathrm{COO}-), 7.46$ (d, $J=3.9 \mathrm{~Hz}, 1 \mathrm{H}$, C3-H), 7.50 (d, $J=10.9 \mathrm{~Hz}, \overline{1} \mathrm{H}, \mathrm{C} 5-\mathrm{H}), 7.61$ (dd, $J=10.9,1.9$ $\mathrm{Hz}, 1 \mathrm{H}, \mathrm{C} 6-\mathrm{H}$ ), 7.74 (d, $J=3.9 \mathrm{~Hz}, 1 \mathrm{H}, \mathrm{C} 2-\mathrm{H}), 8.30$ (d, $J=1.9$ $\mathrm{Hz}, 1 \mathrm{H}, \mathrm{C} 8-\mathrm{H}), 8.51$ (d, $J=15.7 \mathrm{~Hz}, 1 \mathrm{H},-\mathrm{C} \underline{\mathrm{H}}=\mathrm{CH}-\mathrm{COO}-$ ) ppm. ${ }^{13} \mathrm{C}$ NMR $\left(500 \mathrm{MHz},\left(\mathrm{CD}_{3}\right)_{2} \mathrm{CO}\right) \delta 13.0,24.9,32.6$ (br), 39.0, 62.4, 113.0, 121.2, 122.5, 127.1, 134.2, 135.7, 138.0, 138.4, 138.8, 140.1, 142.2, 143.7, 166.7 (br) ppm. IR $\nu_{\max } 977,1001$, 1378, 1414, 1652, $2958 \mathrm{~cm}^{-1}$. HRMS $\left(\mathrm{ESI}^{+}\right)$: calc. for $\mathrm{C}_{19} \mathrm{H}_{23} \mathrm{NO}_{2}[\mathrm{M}+\mathrm{H}]^{+}$298.1810, found 298.1812.

(Z)-N-tert-Butyl-1-(7-isopropyl-1-methylazulen-4-yl)methanimine oxide (23). To aldehyde 11 (70 mg, $0.3 \mathrm{mmol}, 1.0$ eq.) was added $N$-tert-butylhydroxylamine hydrochloride $(62 \mathrm{mg}$, 
$0.5 \mathrm{mmol}, 1.5$ eq.) and pyridine ( $5 \mathrm{~mL}$ ) under nitrogen. The mixture was stirred at $95{ }^{\circ} \mathrm{C}$ until all the starting material was consumed by TLC $(3 \mathrm{~h})$. After cooling to $\mathrm{rt}$, the pyridine was evaporated under reduced pressure. The mixture was diluted with water $(10 \mathrm{~mL})$ and extracted with EtOAc $(3 \times 30 \mathrm{~mL})$ and the combined organic fractions were washed with brine and dried over $\mathrm{MgSO}_{4}$ and filtered. The filtrate was evaporated under reduced pressure to yield a turquoise/green waxy gum (64 mg, 69\%). ${ }^{1} \mathrm{H}$ NMR $\left(500 \mathrm{MHz},\left(\mathrm{CDCl}_{3}\right) \delta 1.38(\mathrm{~d}, J=6.9 \mathrm{~Hz}\right.$, $\left.6 \mathrm{H},-\mathrm{CH}\left(\mathrm{CH}_{3}\right)_{2}\right), 1.71\left(\mathrm{~s}, 9 \mathrm{H}, \mathrm{C}\left(\mathrm{CH}_{3}\right)_{3}\right), 2.68\left(\mathrm{~s}, 3 \mathrm{H}, \mathrm{C} 1-\mathrm{CH}_{3}\right)$, 3.12 (hept, $\left.J=6.9 \mathrm{~Hz}, 1 \mathrm{H},-\mathrm{CH}\left(\mathrm{CH}_{3}\right)_{2}\right), 7.18(\mathrm{~d}, J=3.7 \mathrm{~Hz}, 1 \mathrm{H}$, C3-H), 7.57 (dd, $J=11.0,2.0 \mathrm{~Hz}, 1 \mathrm{H}, \mathrm{C} 6-\mathrm{H}), 7.67$ (d, $J=3.7 \mathrm{~Hz}$, $1 \mathrm{H}, \mathrm{C} 2-\mathrm{H}), 8.21$ (d, $J=1.8 \mathrm{~Hz}, 1 \mathrm{H}, \mathrm{C} 8-\mathrm{H}), 8.37$ (s, $1 \mathrm{H}$, $-\mathrm{CH}=\mathrm{N}), 8.85(\mathrm{~d}, J=11.0 \mathrm{~Hz}, \mathrm{C} 5-\mathrm{H}) \mathrm{ppm} .{ }^{13} \mathrm{C}$ NMR $(500 \mathrm{MHz}$, $\left(\mathrm{CDCl}_{3}\right) \delta 13.1,24.7,28.6,38.7,72.6,109.7,121.5,125.4,129.5$, $133.5,134.0,135.3,137.1,137.4,138.4,143.3 \mathrm{ppm}$. IR $\nu_{\max }$ 766, 1131, 1361, 1537, $2958 \mathrm{~cm}^{-1}$. HRMS (ESI ${ }^{+}$): calc. for $\mathrm{C}_{19} \mathrm{H}_{25} \mathrm{NO}[\mathrm{M}+\mathrm{H}]^{+}$284.2009, found 284.2019.

\section{Conflicts of interest}

There are no conflicts to declare.

\section{Acknowledgements}

We thank the University of Bath for funding. TDJ wishes to thank the Royal Society for a Wolfson Research Merit Award and the Open Research Fund of School of Chemistry and Chemical Engineering, Henan Normal University for support (2020ZD01).

\section{Notes and references}

1 R. S. H. Liu, J. Chem. Educ., 2002, 79, 183.

2 (a) M. Beer and H. C. Longuet-Higgins, J. Chem. Phys., 1955, 23, 1390; (b) B. D. Wagner, D. Tittelbach-Helmrich and R. P. Steer, J. Phys. Chem., 1992, 96, 7904; (c) K. Veys and D. Escudero, J. Phys. Chem. A, 2020, 124, 7228.

3 (a) T. Shoji, S. Sugiyama, Y. Kobayashi, A. Yamazaki, Y. Ariga, R. Katoh, H. Wakui, M. Yasunami and S. Ito, Chem. Commun., 2020, 56, 1485; (b) P. Dunås, L. C. Murfin, O. J. Nilsson, N. Jame, S. E. Lewis and N. Kann, J. Org. Chem., 2020, 85, 13453; (c) K. Ohtsu, R. Hayami, T. Sagawa, S. Tsukada, K. Yamamoto and T. Gunji, Tetrahedron, 2019, 75, 130658; (d) S. J. Webster, C. M. López-Alled, X. Liang, C. L. McMullin, G. Kociok-Köhn, C. L. Lyall, T. D. James, J. Wenk, P. J. Cameron and S. E. Lewis, New J. Chem., 2019, 43, 992; (e) H. L. Gao, X. D. Yang, H. S. Xin, T. Z. Gao, H. G. Gong and X. K. Gao, Chin. J. Org. Chem., 2018, 38, 2680; $(f)$ T. Tang, T. T. Lin, F. Erden, F. Wang and C. B. He, J. Mater. Chem. C, 2018, 6, 5153; $(g)$ L. Z. Gai, J. Z. Chen, Y. Zhao, J. Mack, H. Lu and Z. Shen, RSC Adv., 2016, 6, 32124; (h) E. H. Ghazvini Zadeh, S. Tang, A. W. Woodward,
T. Liu, M. V. Bondar and K. D. Belfield, J. Mater. Chem. C, 2015, 3, 8495; (i) M. Murai, K. Takami, H. Takeshima and K. Takai, Org. Lett., 2015, 17, 1798; (j) E. Amir, M. Murai, R. J. Amir, J. S. Cowart, M. L. Chabinyc and C. J. Hawker, Chem. Sci., 2014, 5, 4483; (k) M. Murai, S. Y. Ku, N. D. Treat, M. J. Robb, M. L. Chabinyc and C. J. Hawker, Chem. Sci., 2014, 5, 3753; (l) M. Koch, O. Blacque and K. Venkatesan, J. Mater. Chem. C, 2013, 1, 7400; (m) M. Koch, O. Blacque and K. Venkatesan, Org. Lett., 2012, 14, 1580; (n) E. Amir, R. J. Amir, L. M. Campos and C. J. Hawker, J. Am. Chem. Soc., 2011, 133, 10046.

4 (a) T. Shoji and S. Ito, Chem. - Eur. J., 2017, 23, 16696; (b) T. Shoji, M. Maruyama, A. Maruyama, S. Ito, T. Okujima and K. Toyota, Chem. - Eur. J., 2014, 20, 11903; (c) T. Shoji, A. Yamamoto, Y. Inoue, E. Shimomura, S. Ito, J. Higashi and N. Morita, Chem. Lett., 2012, 41, 1644; (d) A. C. Razus, L. Birzan, N. M. Surugiu, A. C. Corbu and F. Chiraleu, Dyes Pigm., 2007, 74, 26; (e) A. Blice-Baum, A. Van Dyke, I. Sigmon, E. A. Salter, A. Wierzbicki, Y. Pocker and G. T. Spyridis, Int. J. Quantum Chem., 2006, 106, 2331; (f) L. Cristian, I. Sasaki, P. G. Lacroix, B. Donnadieu, I. Asselberghs, K. Clays and A. C. Razus, Chem. Mater., 2004, 16, 3543; (g) A. C. Razus, L. Birzan, S. Nae, L. Cristian, F. Chiraleu and V. Cimpeanu, Dyes Pigm., 2003, 57, 223; (h) S. Ito, S. Kikuchi, H. Kobayashi, N. Morita and T. Asao, J. Org. Chem., 1997, 62, 2423.

5 (a) M. Okamoto, S. Hirayama and R. P. Steer, Can. J. Chem., 2007, 85, 432; (b) S. Hirayama and R. P. Steer, J. Chem. Educ., 2008, 85, 317.

6 A. G. Anderson Jr. and B. M. Steckler, J. Am. Chem. Soc., 1959, 81, 4941.

7 (a) H. Xin, J. Li, X. Yang and X. Gao, J. Org. Chem., 2020, 85, 70; (b) L. C. Murfin, C. M. López-Alled, A. C. Segwick, J. Wenk, T. D. James and S. E. Lewis, Front. Chem. Sci. Eng., 2020, 14, 90; (c) C. M. López-Alled, L. C. Murfin, G. KociokKöhn, T. D. James, J. Wenk and S. E. Lewis, Analyst, 2020, 145, 6262; (d) L. C. Murfin, K. Chiang, G. T. Williams, C. L. Lyall, A. T. A. Jenkins, J. Wenk, T. D. James and S. E. Lewis, Front. Chem., 2020, 8, 10; (e) H. Gao, C. Ge, B. Hou, H. Xin and X. Gao, ACS Macro Lett., 2019, 8, 1360; (f) H. Xin, J. Li, X. Yang and X. Gao, J. Org. Chem., 2019, 85, 70; $(g)$ D. Lichosyt, S. Wasiłek, P. Dydio and J. Jurczak, Chem. Eur. J., 2018, 24, 11683; (h) G.-O. Buica, I.-G. Lazar, L. Birzan, C. Lete, M. Prodana, M. Enachescu, V. Tecuceanu, A. B. Stoian and E.-M. Ungureanu, Electrochim. Acta, 2018, 263, 382; (i) C. M. López-Alled, A. Sanchez-Fernandez, K. J. Edler, A. C. Sedgwick, S. D. Bull, C. L. McMullin, G. Kociok-Köhn, T. D. James, J. Wenk and S. E. Lewis, Chem. Commun., 2017, 53, 12580; (j) L. Birzan, M. Cristea, C. C. Draghici, V. Tecuceanu, M. Maganu, A. Hanganu, G.-L. Arnold, E.-M. Ungureanu and A. C. Razus, Tetrahedron, 2016, 72, 2316; (k) S. Wakabayashi, M. Uchida, R. Tanaka, Y. Habata and M. Shimizu, Asian J. Org. Chem., 2013, 2, 786.

8 (a) L. C. Murfin and S. E. Lewis, Molecules, 2021, 26, 353; (b) L. C. Murfin, M. Weber, S. J. Park, W. T. Kim, 
C. M. Lopez-Alled, C. L. McMullin, F. Pradaux-Caggiano, C. L. Lyall, G. Kociok-Köhn, J. Wenk, S. D. Bull, J. Yoon, H. M. Kim, T. D. James and S. E. Lewis, J. Am. Chem. Soc., 2019, 141, 19389; (c) Y. Zhou, G. Baryshnikov, X. Li, M. Zhu, H. Ågren and L. Zhu, Chem. Mater., 2018, 30, 8008; (d) P. M. Gosavi, Y. S. Moroz and I. V. Korendovych, Chem. Commun., 2015, 51, 5347; (e) Y. S. Moroz, W. Binder, P. Nygren, G. A. Caputo and I. V. Korendovych, Chem. Commun., 2013, 49, 490; (f) H. Salman, Y. Abraham, S. Tal, S. Meltzman, M. Kapon, N. Tessler, S. Speiser and Y. Eichen, Eur. J. Org. Chem., 2005, 2207; (g) C. Mazzuca, L. Stella, M. Venanzi, F. Formaggio, C. Toniolo and B. Pispisa, Biophys. J., 2005, 88, 3411; (h) G. Loidl, H.-J. Musiol, N. Budisa, R. Huber, S. Poirot, D. Fourmy and L. Moroder, J. Pept. Sci., 2000, 6, 139.

9 (a) L. Yang, Y. Zhu, J. Liu, Y. Chen, J. Wu, Z. Pang, Z. Lu, S. Zhao and Y. Huang, Dyes Pigm., 2021, 187, 109079; (b) P. Cowper, A. Pockett, G. Kociok-Köhn, P. J. Cameron and S. E. Lewis, Tetrahedron, 2018, 74, 2775; (c) H. Xin, C. Ge, X. Jiao, X. Yang, K. Rundel, C. R. McNeill and X. Gao, Angew. Chem., Int. Ed., 2018, 57, 1322; (d) E. Puodziukynaite, H.-W. Wang, J. Lawrence, A. J. Wise, T. P. Russell, M. D. Barnes and T. Emrick, J. Am. Chem. Soc., 2014, 136, 11043; (e) K. Kakiage, E. Fujimura, Y. Nakada, T. Ogino, T. Kyomen and M. Hanaya, Key Eng. Mater., 2014, 596, 35; (f) X.-H. Zhang, C. Li, W.-B. Wang, X.-X. Cheng, X.-S. Wang and B.-W. Zhang, J. Mater. Chem., 2007, 17, 642.

10 (a) B. Hou, J. Li, H. Xin, X. Yang, H. Gao, P. Peng and X. Gao, Acta Chim. Sin., 2020, 78, 788; (b) Y. Shibuya, K. Aonuma, T. Kimura, T. Kaneko, W. Fujiwara, Y. Yamaguchi, D. Kumaki, S. Tokito and H. Katagiri, J. Phys. Chem. C, 2020, 124, 4738; (c) H. Xin, J. Li, R.-Q. Lu, X. Gao and T. M. Swager, J. Am. Chem. Soc., 2020, 142, 13598; (d) H. Gao, C. Ge, B. Hou, H. Xin and X. Gao, ACS Macro Lett., 2019, 8, 1360; (e) H. Xin, J. Li, C. Ge, X. Yang, T. Xue and X. Gao, Mater. Chem. Front., 2018, 2, 975; (f) H. Xin, C. Ge, L. Fu, X. Yang and X. Gao, Chin. J. Org. Chem., 2017, 37, 711; $(g)$ H. Xin and X. Gao, ChemPlusChem, 2017, 82, 945; (h) Y. Yamaguchi, M. Takubo, K. Ogawa, K.-i. Nakayama, T. Koganezawa and H. Katagiri, J. Am. Chem. Soc., 2016, 138, 11335; (i) H. Xin, C. Ge, X. Yang, H. Gao, X. Yang and X. Gao, Chem. Sci., 2016, 7, 6701; (j) H. Nishimura, N. Ishida, A. Shimazaki, A. Wakamiya, A. Saeki, L. T. Scott and Y. Murata, J. Am. Chem. Soc., 2015, 137, 15656; (k) J. Yao, Z. Cai, Z. Liu, C. Yu, H. Luo, Y. Yang, S. Yang, G. Zhang and D. Zhang, Macromolecules, 2015, 48, 2039; (l) J. Xia, B. Capozzi, S. Wei, M. Strange, A. Batra, J. R. Moreno, R. J. Amir, E. Amir, G. C. Solomon, L. Venkataraman and L. M. Campos, Nano Lett., 2014, 14, 2941; (m) Y. Yamaguchi, K. Ogawa, K. Nakayama, Y. Ohba and H. Katagiri, J. Am. Chem. Soc., 2013, 135, 19095; (n) Y. Yamaguchi, Y. Maruya, H. Katagiri, K.-i. Nakayama and Y. Ohba, Org. Lett., 2012, 14, 2316.
11 (a) A. Turku, T. O. Leino, L. Karhu, J. Yli-Kauhaluoma, J. P. Kukkonen, E. A. A. Wallén and H. Xhaard, ChemMedChem, 2019, 14, 965; (b) T. Wada, R. Maruyama, Y. Irie, M. Hashimoto, H. Wakabayashi, N. Okudaira, Y. Uesawa, H. Kagaya and H. Sakagami, In Vivo, 2018, 32, 479; (c) T. O. Leino, A. Turku, J. Yli-Kauhaluoma, J. P. Kukkonen, H. Xhaard and E. A. A. Wallén, Eur. J. Med. Chem., 2018, 157, 88; (d) E. Stempel, R. F.-X. Kaml, N. Budisa and M. Kalesse, Bioorg. Med. Chem., 2018, 26, 5259; (e) J. Peet, A. Selyutina and A. Bredihhin, Bioorg. Med. Chem., 2016, 24, 1653; (f) K. Ikegai, M. Imamura, T. Suzuki, K. Nakanishi, T. Murakami, E. Kurosaki, A. Noda, Y. Kobayashi, M. Yokota, T. Koide, K. Kosakai, Y. Okhura, M. Takeuchi, H. Tomiyama and M. Ohta, Bioorg. Med. Chem., 2013, 21, 3934; (g) S. Löber, H. Hübner, A. Buschauer, F. Sanna, A. Argiolas, M. R. Melis and P. Gmeiner, Bioorg. Med. Chem. Lett., 2012, 22, 7151; (h) C. Chen, O. Lee, C. Yao, M. Chuang, Y. Chang, M. Chang, Y. Wen, W. Yang, C. Ko, N. Chou, M. Lin, C. Lai, C. Sun, L. Wang, Y. Chen, T. Hseu, C. Chang, H. Hsu, H. Lin, Y. Chang, Y. Shih, S. Chou, Y. Hsu, H. Tseng, C. Liu, C. Tu, T. Hu, Y. Tsai, T. Chen, C. Lin, S. Chiou, C. Liu and C. Hwang, Bioorg. Med. Chem. Lett., 2010, 20, 6129; (i) Y. Tanaka and K. Shigenobu, Cardiovasc. Drug Rev., 2001, 19, 297; (j) A. E. Asato, A. Peng, M. Z. Hossain, T. Mirzadegan and J. S. Bertram, J. Med. Chem., 1993, 36, 3137; (k) T. Tomiyama, M. Yokota, S. Wakabayashi, K. Kosakai and T. Yanagisawa, J. Med. Chem., 1993, 36, 791; (l) T. Yanagisawa, S. Wakabayashi, T. Tomiyama, M. Yasunami and K. Takase, Chem. Pharm. Bull., 1988, 36, 641.

12 For recent reviews, see: (a) A. Konishi and M. Yasuda, Chem. Lett., 2021, 50, 195; (b) T. Shoji, T. Okujima and S. Ito, Int. J. Mol. Sci., 2020, 21, 7087; (c) H. N. Zeng, Z. M. Png and J. Xu, Chem. - Eur. J., 2020, 15, 1904; (d) A. C. Razus and L. Birzan, Monatsh. Chem., 2019, 150, 139; (e) T. Shoji and S. Ito, Adv. Heterocycl. Chem., 2018, 126, 1.

13 (a) J. Mogensen, O. Christensen, M. D. Kilde, M. Abildgaard, L. Metz, A. Kadziola, M. Jevric, K. V. Mikkelsen and M. B. Nielsen, Eur. J. Org. Chem., 2019, 1986; (b) M. D. Kilde, M. H. Hansen, S. L. Broman, K. V. Mikkelsen and M. B. Nielsen, Eur. J. Org. Chem., 2017, 1052; (c) A. U. Petersen, M. Jevric, R. J. Mandle, M. T. Sims, J. N. Moore, S. J. Cowling, J. W. Goodby and M. B. Nielsen, Chem. - Eur. J., 2017, 23, 5090; (d) A. U. Petersen, M. Jevric, J. Elm, S. T. Olsen, C. G. Tortzen, A. Kadziola, K. V. Mikkelsen and M. B. Nielsen, Org. Biomol. Chem., 2016, 14, 2403; (e) A. Vlasceanu, C. L. Andersen, C. R. Parker, O. Hammerich, T. J. Morsing, M. Jevric, S. L. Broman, A. Kadziola and M. B. Nielsen, Chem. - Eur. J., 2016, 22, 7514; (f) A. B. Skov, S. L. Broman, A. S. Gertsen, J. Elm, M. Jevric, M. Cacciarini, A. Kadziola, K. V. Mikkelsen and M. B. Nielsen, Chem. - Eur. J., 2016, 22, 14567; $(g)$ M. D. Kilde, S. L. Broman, A. Kadziola and M. B. Nielsen, Synlett, 2016, 450; (h) S. L. Broman, 
O. Kushnir, M. Rosenberg, A. Kadziola, J. Daub and M. B. Nielsen, Eur. J. Org. Chem., 2015, 4119; (i) S. L. Broman, M. Jevric, A. D. Bond and M. B. Nielsen, J. Org. Chem., 2014, 79, 41; (j) M. Jevric, S. L. Broman and M. B. Nielsen, J. Org. Chem., 2013, 78, 4348; (k) S. L. Broman, S. Lara-Avila, C. L. Thisted, A. D. Bond, S. Kubatkin, A. Danilov and M. B. Nielsen, Adv. Funct. Mater., 2012, 22, 4249; (l) V. Mazzanti, M. Cacciarini, S. L. Broman, C. R. Parker, M. Schau-Magnussen, A. D. Bond and M. B. Nielsen, Beilstein J. Org. Chem., 2012, 8, 958; (m) M. Å. Petersen, A. S. Andersson, K. Kilså and M. B. Nielsen, Eur. J. Org. Chem., 2009, 1855.

14 (a) A. L. Crombie, J. L. Kane, K. M. Shea and R. L. Danheiser, J. Org. Chem., 2004, 69, 8652; (b) J. L. Kane, K. M. Shea, A. L. Crombie and R. L. Danheiser, Org. Lett., 2001, 3, 1081.

15 (a) A. G. Anderson Jr., J. A. Nelson and J. J. Tazuma, J. Am. Chem. Soc., 1953, 75, 4980; (b) A. G. Anderson Jr., R. Scotoni Jr., E. J. Cowles and C. G. Fritz, J. Org. Chem., 1957, 22, 1193; (c) A. G. Anderson Jr., R. G. Anderson and L. L. Replogle, Proc. Chem. Soc., 1960, 72; (d) A. G. Anderson Jr. and R. G. Anderson, J. Org. Chem., 1962, 27, 3578; (e) L. J. Mathias and C. G. Overberger, J. Org. Chem., 1980, 45, 1701.

16 (a) P. Cowper, Y. Jin, M. D. Turton, G. Kociok-Köhn and S. E. Lewis, Angew. Chem., Int. Ed., 2016, 55, 2564; (b) J. Dubovik and A. Bredihhin, Synthesis, 2015, 538; (c) J. Dubovik and A. Bredihhin, Synthesis, 2015, 2663; (d) S. Ito, T. Shoji and N. Morita, Synlett, 2011, 2279; (e) T. Shoji, S. Ito, K. Toyota, T. Iwamoto, M. Yasunami and N. Morita, Eur. J. Org. Chem., 2009, 4307; (f) M. Fujinaga, K. Suetake, K. Gyoji, T. Murafuji, K. Kurotobi and Y. Sugihara, Synthesis, 2008, 3745; (g) T. Shibasaki, T. Ooishi, N. Yamanouchi, T. Murafuji, K. Kurotobi and Y. Sugihara, J. Org. Chem., 2008, 73, 7971; (h) S. Ito, T. Terazono, T. Kubo, T. Okujima, N. Morita, T. Murafuji, Y. Sugihara, K. Fujimori, J. Kawakami and A. Tajiri, Tetrahedron, 2004, 60, 5357.

17 (a) S. C. Lee, J.-Y. Son, J. Y. Kim, H. Eom, S. B. Jang and P. H. Lee, Adv. Synth. Catal., 2021, 363, 512; (b) Z. M. Png, T. L. D. Tam and J. Xu, Org. Lett., 2020, 22, 5009; (c) Z. M. Png and J. Xu, Chem. - Asian J., 2020, 15, 2505; (d) B. Pigulski, K. Shoyama and F. Würthner, Angew. Chem., 2020, 59, 15908; (e) C. Maeng, H. J. Seo, H. Jeong, K. Lee, H. C. Noh and P. H. Lee, Org. Lett., 2020, 22, 7267; (f) C. Maeng, J.-Y. Son, S. C. Lee, Y. Baek, K. Um, S. H. Han, G. H. Ko, G. U. Han, K. Lee, K. Lee and P. H. Lee, J. Org. Chem., 2020, 85, 3824; $(g)$ G. U. Han, J.-Y. Son, D. Park, H. Eom, K. Lee, H. C. Noh, K. Lee and P. H. Lee, Adv. Synth. Catal., 2020, 362, 4749; (h) X. Shi, A. Sasmal, J.-F. Soulé and H. Doucet, Chem. - Asian J., 2018, 13, 143; (i) J. Carreras, Y. Popowski, A. Caballero, E. Amir and P. J. Perez, J. Org. Chem., 2018, 83, 11125; (j) A. Székely, A. Péter, K. Aradi, G. L. Tolnai and Z. Novák, Org. Lett., 2017, 19, 954; (k) M. Murai, S. Iba, H. Ota and K. Takai, Org. Lett., 2017, 19, 5585; (l) M. Murai,
M. Yanagawa, M. Nakamura and K. Takai, Asian J. Org. Chem., 2016, 5, 629; (m) M. Murai, K. Takami, H. Takeshima and K. Takai, Org. Lett., 2015, 17, 1798; (n) L. Zhao, C. Bruneau and H. Doucet, Chem. Commun., 2013, 49, 5598; (o) M. Fujinaga, T. Murafuji, K. Kurotobi and Y. Sugihara, Tetrahedron, 2009, 65, 7115; ( $p$ ) J. Liu, E. Muth, U. Floerke, G. Henkel, K. Merz, J. Sauvageau, E. Schwake and G. Dyker, Adv. Synth. Catal., 2006, 348, 456; (q) K. Kurotobi, M. Miyauchi, K. Takakura, T. Murafuji and Y. Sugihara, Eur. J. Org. Chem., 2003, 3663; (r) G. Dyker, S. Borowski, J. Heiermann, J. Körning, K. Opwis, G. Henkel and M. Köckerling, J. Organomet. Chem., 2000, 606, 108.

18 (a) M. Makosza, P. W. Osinski and S. Ostrowski, Pol. J. Chem., 2001, 75, 275; (b) M. Makosza and R. Podraza, Eur. J. Org. Chem., 2000, 193.

19 (a) T. Morita, N. Abe and K. Takase, J. Chem. Soc., Perkin Trans. 1, 2000, 3063; (b) R. N. McDonald, H. E. Petty, N. L. Wolfe and J. V. Paukstelis, J. Org. Chem., 1974, 39, 1877; (c) K. Hafner and H. Weldes, Liebigs Ann., 1957, 606, 90.

20 (a) D. Copland, D. Leaver and W. B. Menzies, Tetrahedron Lett., 1977, 18, 639; (b) S. E. Reiter, L. C. Dunn and K. N. Houk, J. Am. Chem. Soc., 1977, 99, 4199; (c) L. T. Scott, M. A. Minton and M. A. Kirms, J. Am. Chem. Soc., 1980, 102, 6311; (d) H. Langhals and M. Eberspächer, Synthesis, 2018, 1862.

21 See: D. Chen, S. Yu, L. van Ofwegen, P. Proksch and W. Lin, J. Agric. Food Chem., 2012, 60, 112 and references therein.

22 G. Li, Chin. Pat, CN109096038A, 2018.

23 Y. Zhou, Chin. Pat, CN105753623A, 2016.

24 F. Xu, Chin. Pat, CN108863707A, 2018.

25 See: T. Yanagisawa, K. Kosakai, T. Tomiyama, M. Yasunami and K. Takase, Chem. Pharm. Bull., 1990, 38, 3355 and references therein.

26 Y. Inoue, I. Shinohara, I. Murata and I. Kanamoto, J. Mol. Struct., 2019, 1186, 50.

27 Y. Matsubara, H. Yamamoto and T. Nozoe, Stud. Nat. Prod. Chem., 1994, 14, 313.

28 (a) T. Nozoe, S. Ishikawa and K. Shindo, Chem. Lett., 1989, 18, 353; (b) Z. Zhao, Y. Matsubara and T. Nozoe, Nippon Kagaku Kaishi, 1994, 49, DOI: 10.1246/nikkashi.1994.49.

29 (a) J. Richter, P. Liebing and F. T. Edelmann, Inorg. Chim. Acta, 2018, 475, 18; (b) A. Kiriazis, I. B. Aumüller, R. Arnaudova, V. Brito, T. Rüffer, H. Lang, S. M. Silvestre, P. J. Koskinen and J. Yli-Kauhaluoma, Org. Lett., 2017, 19, 2030; (c) A. W. Woodward, E. H. Ghazvini Zadeh, M. V. Bondar and K. D. Belfield, R. Soc. Open Sci., 2016, 3, 160373; (d) O. Sato, T. Saito, M. Iwase and A. Sakai, Heterocycles, 2016, 93, 714; (e) L. Birzan, M. Cristea, C. C. Draghici, V. Tecuceanu, M. Maganu, A. Hanganu, G.-L. Arnold, E.-M. Ungureanu and A. C. Razus, Tetrahedron, 2016, 72, 2316; ( $f$ ) I. B. Aumüller and J. YliKauhaluoma, Org. Lett., 2011, 13, 1670; $(g)$ A. Kiriazis, I. B. Aumüller and J. Yli-Kauhaluoma, Tetrahedron Lett., 2011, 52, 1151; (h) R. Sigrist and H.-J. Hansen, Helv. Chim. Acta, 2010, 93, 1545; (i) I. B. Aumüller and T. K. Lindhorst, 
J. Carbohydr. Chem., 2009, 28, 330; (j) A. C. Razus, L. Birzan, V. Tecuceanu, M. Cristea and C. Enache, ARKIVOC, 2008, 210; (k) I. B. Aumüller and T. K. Lindhorst, Eur. J. Org. Chem., 2006, 1103; (l) S. Balduzzi, H. MullerBunz and M. A. McGlinchey, Chem. - Eur. J., 2004, 10, 5398; (m) J. Song and H.-J. Hansen, Helv. Chim. Acta, 1999, 82, 309; (n) A. A. S. Briquet and H.-J. Hansen, Helv. Chim. Acta, 1994, 77, 1921; (o) S. Kurokawa, Nippon Kagaku Kaishi, 1997, 365, DOI: 10.1246/nikkashi.1997.365; (p) S. Kurokawa, Nippon Kagaku Kaishi, 1995, 39, DOI: 10.1246/nikkashi.1995.39; (q) S. Kurokawa, Bull. Chem. Soc. Jpn., 1983, 56, 2311; (r) M. Scholz, L. N. Vien, G. Fischer, B. Tschapke and M. Mühlstädt, Chem. Ber., 1967, 100, 375.

30 (a) R. F. Abdulla and R. S. Brinkmeyer, Tetrahedron, 1979, 35, 1675; (b) F. A. Abu-Shanab, S. M. Sherif and S. A. S. Mousa, J. Heterocycl. Chem., 2009, 46, 801; (c) S. M. Riyadh, I. A. Abdelhamid, H. M. Al-Matar, N. M. Hilmy and M. H. Elnagdi, Heterocycles, 2008, 75, 1849.

31 H. Meerwein, W. Florian, N. Schön and G. Stopp, Liebigs Ann., 1961, 641, 1.

32 (a) H. Bredereck and G. Simchen, Angew. Chem., Int. Ed. Engl., 1963, 2, 738; (b) H. Bredereck, G. Simchen and R. Wahl, Chem. Ber., 1968, 101, 4048.

33 M. G. Vetelino and J. W. Coe, Tetrahedron Lett., 1994, 35, 219.

34 K. Kurotobi, K. S. Kim, S. B. Noh, D. Kim and A. Osuka, Angew. Chem., Int. Ed., 2006, 45, 3944.
35 T. O. Leino, M. Baumann, J. Yli-Kauhaluoma, I. R. Baxendale and E. A. A. Wallén, J. Org. Chem., 2015, 80, 11513.

36 T. Shoji, T. Araki, N. Iida, Y. Kobayashi, A. Ohta, R. Sekiguchi, S. Ito, S. Mori, T. Okujima and M. Yasunami, Eur. J. Org. Chem., 2018, 1145.

37 (a) L. Horner, H. Hoffmann and H. G. Wippel, Chem. Ber., 1958, 91, 61; (b) W. S. Wadsworth Jr. and W. D. Emmons, J. Am. Chem. Soc., 1961, 83, 1733.

38 M. A. Blanchette, W. Choy, J. T. Davis, A. P. Essenfeld, S. Masamune, W. R. Roush and T. Sakai, Tetrahedron Lett., 1984, 25, 2183.

39 (a) S. Itoh, N. Morita, R. Miyatake and S. Kuroda, Chem. Lett., 1997, 1011; (b) M. Oda, A. Sakamoto, T. Uchiyama, T. Kajioka, R. Miyatake and S. Kuroda, Tetrahedron Lett., 1999, 40, 3595; (c) M. Oda, H. Kainuma, T. Uchiyama, R. Miyatake and S. Kuroda, Tetrahedron, 2003, 59, 2831; (d) M. Oda, N. Nakajima, N. C. Thanh, T. Kajioka and S. Kuroda, Tetrahedron, 2006, 62, 8177; (e) M. Oda, N. Nakajima, N. C. Thanh, K. Kitahara, R. Miyatake and S. Kuroda, Eur. J. Org. Chem., 2008, 5301.

40 (a) M. Rosselin, B. Poeggeler and G. Durand, Curr. Top. Med. Chem., 2017, 17, 2006; (b) D. A. Becker, J. J. Ley, L. Echegoyen and R. Alvarado, J. Am. Chem. Soc., 2002, 124, 4678; (c) D. A. Becker, J. Am. Chem. Soc., 1996, 118, 905.

41 (a) K. Satoh, M. Yamaguchi and I. Ogura, Bunseki Kagaku, 1983, 32, 61, DOI: 10.2116/bunsekikagaku.32.61; (b) K. Satoh, M. Yamaguchi and I. Ogura, Nippon Kagaku Kaishi, 1982, 1982, 1199, DOI: 10.1246/nikkashi.1982.1199. 